\title{
CÁLCULO DE LAS FRECUENCIAS DE CORTE EN FIBRAS ÓPTICAS DE ÍNDICE ESCALONADO, UTILIZANDO MATLAB
}

\section{A CALCULATION OF CUTOFF FREQUENCY TO STEP-INDEX OPTICAL FIBERS USING MATLAB}

\author{
Henry Arturo Bastidas Mora
}

Ing. Electricista, M.Sc., Docente Investigador, Líder grupo Redes e Interconexión. Programa Ingeniería de Telecomunicaciones. Universidad Piloto de Colombia, Bogotá

henry-bastidas@unipiloto.edu.co

Fabián Barahona Varela

Licenciado en Física, estudiante de Ingeniería Electrónica y Telecomunicaciones.

Universidad Católica de Colombia, Bogotá

fbarahona46@ucatolica.edu.co

Fecha de recepción: 5 de julio de 2012

Fecha de aprobación: 2 de diciembre 2012

\section{RESUMEN}

El análisis matemático de los modos de propagación y de otros parámetros importantes de operación de las guías de onda y las fibras ópticas se puede encontrar al resolver las cuatro ecuaciones de Maxwell y satisfacer adecuadamente las condiciones de frontera en cada caso analizado de acuerdo con su geometría y los materiales con los cuales esté constituido. Sin embargo, estas soluciones resultan muy laboriosas y a veces confusas para los estudiantes que las enfrentan por primera vez. En el presente trabajo se desarrolló e implementó una aplicación utilizando la herramienta Matlab que facilita a los estudiantes y docentes, el procedimiento y los cálculos que se llevan a cabo para obtener algunos parámetros como la apertura numérica, frecuencia de corte normalizada y frecuencia de corte normal para las fibras ópticas de índice escalonado, partiendo de los índices de refracción y el radio del núcleo.

Palabras clave: apertura numérica, fibra óptica, frecuencia de corte normalizada, frecuencia de corte real, índice escalonado.

\begin{abstract}
The math analysis of propagation modes and other relevant operating parameters to waveguides and optical fibers can be found by solving the four equations of Maxwell, and also meeting effectively boundary conditions analyzed for each case according to geometry and materials
\end{abstract}


they are produced with. These solutions however are quite difficult and sometimes confusing for students who use them first time. In this paper we developed and implemented an application using Matlab tool, which provides students and teachers the procedure and calculations carried out to get some parameters, such as numerical aperture, normalized cutoff frequency and actual cutoff frequency to step-index optical fibers based on refractive indices and a core radius.

Keywords: numerical aperture, fiber optics, normalized cutoff frequency, actual cutoff frequency, step-index.

\section{INTRODUCCIÓN}

Las fibras ópticas y en general las guías de onda, no pueden transmitir energía electromagnética a frecuencias bajas, a menos que tengan tamaños gigantescos e imprácticos. La transmisión se inicia a partir de una determinada frecuencia, cuyo valor depende de la geometría, tamaño y materiales de la fibra. A esta frecuencia mínima a partir de la cual es posible que la energía se propague, se le denomina frecuencia de corte. Por sus magníficas prestaciones: gran ancho de banda y su consecuente velocidad de transmisión de información, la inmunidad a perturbaciones electromagnéticas, la baja atenuación con poca dependencia de la frecuencia de trabajo, la alta resistencia mecánica, la flexibilidad y ligereza, entre otros factores, han posicionado a la fibra óptica como uno de los medios de transmisión más utilizados en la actualidad en telecomunicaciones [1].

Desde este marco, cobra gran importancia el estudio de las características de propagación que se encuentran en una fibra óptica, ya que es a través de ellas como se podrá establecer las frecuencias de trabajo, los anchos de banda proporcionados, las aperturas numéricas y otras características que proporcionarán un aprovechamiento óptimo de la fibra.

Se pretende aquí, diseñar e implementar un programa que permita calcular estos parámetros específicamente en una fibra óptica de índice escalonado, es decir, en la que posee un índice de refracción constante en toda la sección cilíndrica del núcleo. Con este objetivo, se utilizará la herramienta Matlab, por considerar que ofrece las características necesarias para calcular ecuaciones matemáticas complejas.

\section{METODOLOGÍA}

Se comienza, examinado algunas definiciones y describiendo los tipos de fibra óptica, para seguir con la formulación comúnmente conocida como la ecuación característica. Luego se definen algunos parámetros importantes para su cálculo. La fibra óptica también se denomina guía de onda dieléctrica, porque a cambio de las guías de onda convencionales no están constituidas por un conductor sino que están hechas con material dieléctrico. Geométricamente, las fibras ópticas 
consisten en una barra dieléctrica cilíndrica delgada y larga llamada núcleo, rodeada de una capa concéntrica de otro material dieléctrico llamada revestimiento; son fabricadas con vidrio flexible, puro y transparente, cuya materia prima es el dióxido de silicio (SiO2), obtenido de la arena del mar. La Figura 1 muestra su estructura en su forma más elemental.

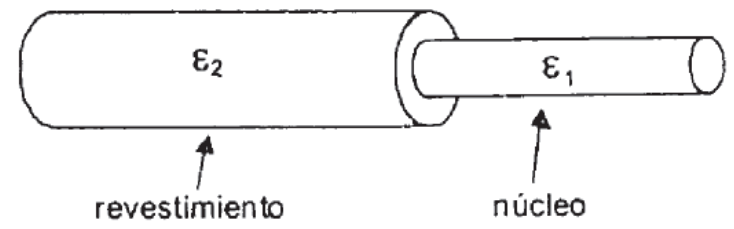

Figura 1. Estructura de una fibra óptica

\subsection{TIPOS DE FIBRA ÓPTICA}

Las fibras ópticas pueden clasificarse en tres tipos: monomodo de índice escalonado, multimodo de índice escalonado y multimodo de índice gradual. La Figura 2 muestra estos tres tipos de fibra y permite comparar el grosor y el índice de refracción del núcleo y revestimiento para cada tipo, así como imaginar la trayectoria de los rayos de luz por los diferentes tipos de fibra.

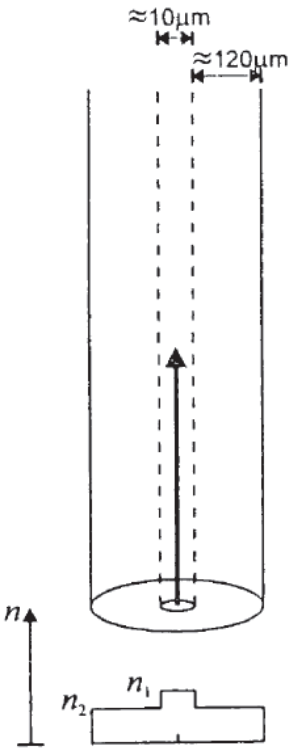

(a)

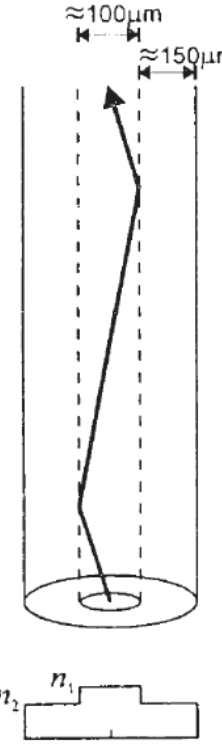

(b)

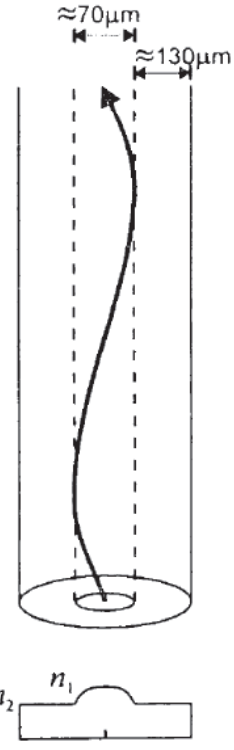

(c)

Figura 2. Comparación del grosor e índice de refracción del núcleo y el revestimiento en fibras a) monomodo de índice escalonado, b) multimodo de índice escalonado, c) multimodo de índice gradual.

Fuente: Vela Nery N., [2] 
La fibra monomodo de índice escalonado tiene un núcleo angosto con índice de refracción uniforme. La fibra multimodo de índice escalonado tiene un núcleo más ancho con índice de refracción constante. La fibra multimodo de índice gradual tiene un núcleo ancho cuyo índice de refracción va variando gradualmente hasta alcanzar su valor máximo en el centro del núcleo.

\subsection{PROPAGACIÓNY ECUACIÓN CARACTERÍSTICA DE UNA FIBRA DE ÍNDICE ESCALONADO}

La Figura 3 es una representación de la fibra de índice escalonado, objeto de estudio, con el sistema de coordenadas cilíndricas que permite formularla.

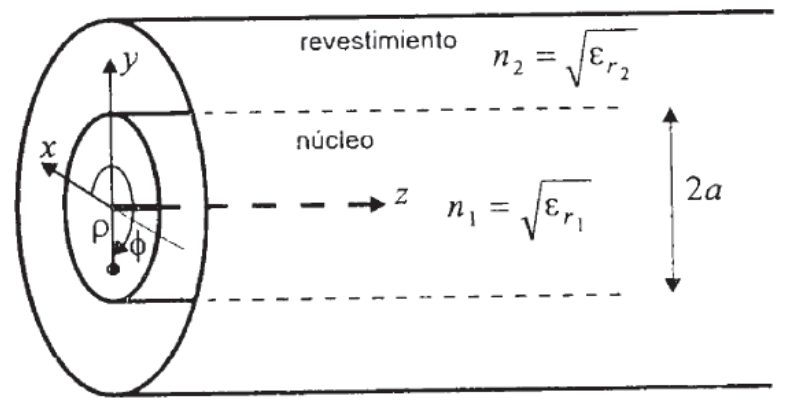

Figura 3. Sistema de coordenadas para analizar la propagación en una fibra óptica con núcleo de radio a

La ecuación diferencial para resolver este tipo de fibra óptica, es idéntica a la que permitiría resolver una guía de onda circular. De conformidad con la formulación corrientemente conocida para guías circulares y de acuerdo con [2] y [3] es:

$$
\frac{1}{\rho} \frac{\partial}{\partial \rho}\left(\rho \frac{\partial \Psi}{\partial \rho}\right)+\frac{1}{\rho^{2}} \frac{\partial^{2} \Psi}{\partial \phi^{2}}+h^{2} \Psi=0
$$

En donde,

$$
h^{2}=\gamma^{2}+\omega^{2} \mu \varepsilon
$$

La variable $\Psi$ puede representar el campo eléctrico $\mathrm{E}_{z}$ o al campo magnético $\mathrm{H}_{z^{\prime}}$ dependiendo de las condiciones de frontera que se empleen en la solución. En principio, en las fibras ópticas, ninguna de las dos componentes $\mathrm{E}_{z} \mathrm{OH}_{z}$ puede valer cero, lo cual origina la aparición de modos de propagación denominados híbridos, designados como HE y EH.

Respecto del núcleo, y tomando en consideración que la permitividad del dieléctrico es constante en toda su trayectoria, la solución de la ec. (1), debe estar en funciones de Bessel de primera clase, así:

$$
\Psi=J_{m}\left(h_{1} \rho\right) \cos m \phi e^{-\gamma z}, \quad(\rho<a)
$$


A partir de la ec. (2), se obtiene:

$$
h_{1}^{2}=\gamma^{2}+\omega^{2} \mu_{0} \varepsilon_{1}, \quad\left(\mu_{r}=1, \varepsilon_{1}=\varepsilon_{0} \varepsilon_{r 1}\right)
$$

Para que la mayor parte de la potencia sea transmitida a lo largo del núcleo, se requiere que la constante $h_{2}{ }^{2}$ sea negativa,

$$
h_{2}{ }^{2}=-\left(\gamma^{2}+\omega^{2} \mu_{0} \varepsilon_{2}\right),\left(\mu_{r}=1, \varepsilon_{2}=\varepsilon_{0} \varepsilon_{r 2}\right)
$$

La solución obtenida para el revestimiento es

$$
\Psi=K_{m}\left(h_{2} \rho\right) \cos m \phi e^{-\gamma z}, \quad(\rho<a)
$$

En donde $K_{m}(h \rho)$ es la función de Bessel modificada de segunda clase, la cual decrece exponencialmente hacia cero mientras $h \rho$ aumenta. Se estima que la propagación en la fibra, se produce a partir de una cierta frecuencia de corte, cuando $\gamma$ se torne imaginaria pura $(\gamma=j \beta)$. Con este objeto, de la ec. (4), se obtiene, con ${h_{1}}^{2}$ positivo, que:

$$
\beta^{2}<\omega^{2} \mu_{0} \varepsilon_{1}
$$

Así mismo, de la ec.(5), con $h_{1}{ }^{2}$ negativo, se obtiene:

$$
\beta^{2}>\omega^{2} \mu_{0} \varepsilon_{2}
$$

Reuniendo las dos expresiones, se obtiene:

$$
\omega^{2} \mu_{0} \varepsilon_{2}<\beta^{2}<\omega^{2} \mu_{0} \varepsilon_{1}
$$

En términos de los índices de refracción del núcleo $n$, y del revestimiento $n_{2}$, teniendo en cuenta que $\varepsilon=n^{2} \varepsilon_{0}$ y que la velocidad de la luz en el vacío equivale a $c=\frac{1}{\sqrt{\mu_{0} \varepsilon_{0}}}$, la ec. (7) puede
escribirse como:

$$
\frac{\omega n_{2}}{c}<\beta<\frac{\omega n_{1}}{c}
$$

Las soluciones generales de $\mathrm{H}_{z}$ tanto para el núcleo como para el revestimiento, se pueden obtener a partir de la ec. (3) y la ec. (6) respectivamente, junto con sus coeficientes. Debe elegirse aquellos que satisfagan las condiciones para la continuidad en la frontera núcleo-revestimiento para toda $z$ en la componente tangencial axial y en la componente tangencial en la dirección $f$ de los campos $\mathrm{E}$ y $\mathrm{H}$. Del mismo modo, las soluciones generales para $\mathrm{E}_{z}$ pueden obtenerse de 
la misma expresión; basta con elegir la función $\operatorname{sen} m \phi$ en lugar de $\cos m \phi$. Realizando un procedimiento matemático similar, a partir de la primera ecuación de Maxwell, puede obtenerse también, las otras componentes para los campos E y H. Un mayor detalle se puede encontrar en [4] y [5].

Dado que la ec. (8) indica el rango de valores para la constante de fase $\beta$, puede procederse a calcularla para cada modo de propagación. Con este objetivo, se plantea la ecuación característica, cuya solución proporciona dicho valor, satisfaciendo las condiciones de continuidad, es decir, que $\mathrm{E}_{\phi}$ y $\mathrm{H}_{\phi}$ deben ser idénticos en ambos dieléctricos para $\rho=a$. Estas dos ecuaciones estarán dadas por:

y

$$
\begin{gathered}
\frac{A \omega \mu}{p} f_{m}(p)-\frac{B m \beta}{p^{2}}=-\frac{A \omega \mu}{q} g_{m}(q)-\frac{B m \beta}{q^{2}} \\
\frac{A m \beta}{p^{2}}-\frac{B \omega \varepsilon_{1}}{p} f_{m}(p)=-\frac{A m \beta}{q^{2}}+\frac{A \omega \varepsilon_{2}}{q} g_{m}(q)
\end{gathered}
$$

Despejando el cociente B/A e igualándolos, teniendo en cuenta que $\mu=\mu_{0} \mu=\mu_{0}$ para los dos dieléctricos, se obtiene la ecuación característica de la fibra

$$
\omega^{2} \mu_{0}\left[\frac{f_{m}(p)}{p}+\frac{g_{m}(q)}{q}\right]\left[\frac{\varepsilon_{1} f_{m}(p)}{p}+\frac{\varepsilon_{2} g_{m}(q)}{q}\right]=m^{2} \beta^{2}\left[\frac{1}{p^{2}}+\frac{1}{q^{2}}\right]
$$

La asignación de los modos de propagación según sus subíndices mn se explica, analizando las funciones de Bessel; $J_{m}$ pasará $n$ veces por el eje de la ordenada igual a cero, por lo cual, existirán $n$ raíces para cada valor de $m$. Las combinaciones posibles de $m$ y $n$ corresponden a un modo de propagación diferente, donde su constante de fase puede designarse como $\beta_{m n}$ y los modos pueden ser $\mathrm{TE}_{m n^{\prime}} \mathrm{TM}_{m n^{\prime}} \mathrm{EH}_{m n^{\prime}} \mathrm{HE}_{m n^{\prime}}$. Para cada caso particular, la ecuación característica (ec.(13)), se simplifica dependiendo de los coeficientes [6].

Frecuencia Normalizada. A partir de la ec. (4) y ec. (5), los valores de p y q deben satisfacer

$$
p^{2}+q^{2}=\omega^{2} \mu_{0}\left(\varepsilon_{1}-\varepsilon_{2}\right) a^{2}
$$

El segundo término de la ecuación se denomina frecuencia normalizada $V$, parámetro Vo número $V$, que está relacionado con la condición de corte de la fibra. $V$ define cuántos modos diferentes puede haber en la fibra simultáneamente a una determinada frecuencia. A partir de la ec.(14), la frecuencia normalizada puede expresarse también como

$$
V^{2}=\omega^{2} \mu_{0} a^{2}\left(\varepsilon_{0} \varepsilon_{r_{1}}-\varepsilon_{0} \varepsilon_{r_{2}}\right)
$$




$$
\begin{array}{r}
V^{2}=\omega^{2} \mu_{0} a^{2}\left(n_{1}^{2}-n_{2}{ }^{2}\right) \\
V^{2}=(2 \pi f)^{2} \frac{a^{2}}{c^{2}}\left(n_{1}{ }^{2}-n_{2}{ }^{2}\right)
\end{array}
$$

Finalmente,

$$
V=\left(\frac{2 \pi a}{\lambda_{0}}\right)^{2}\left(n_{1}{ }^{2}-n_{2}{ }^{2}\right)^{\frac{1}{2}}
$$

Apertura Numérica. La raíz cuadrada de la diferencia de cuadrados de los índices de refracción de la ec. (18), recibe el nombre de Apertura Numérica NA

$$
N A=\sqrt{n_{1}^{2}-n_{2}^{2}}
$$

Constante de fase y frecuencia de corte de los modos en una fibra de índice escalonado

La ec. (13), sólo se puede resolver, utilizando métodos numéricos donde las raíces serán aquellas para las cuales $E_{z}$ y $H_{z}$ no valgan cero (modos híbridos). La solución de esta ecuación es'

$$
\beta=\sqrt{\beta_{0}{ }^{2} n_{1}{ }^{2}-\left(\frac{p}{a}\right)^{2}}
$$

Los subíndices de cada modo nm se asocian con la condición de corte. Puede notarse que el modo $\mathrm{HE}_{11}$ no posee frecuencia de corte, ya que existe desde la frecuencia cero. Los modos restantes poseen valor de corte cuando $\beta / \beta_{0}=n_{2}$, por lo cual, los primeros en producirse después de $\mathrm{HE}_{11}$, son los $\mathrm{TE}_{01}$ y $T \mathrm{TM}_{01}$, cuando $\mathrm{V}=2.405$. Por esta razón, el modo dominante es $\mathrm{HE}_{11}$ que será el único modo en la fibra, siempre y cuando $V<2.405$.

Los demás valores que definen la condición de corte para los demás modos de propagación, así como la nomenclatura a partir del subíndice $\mathrm{mn}$, se obtienen al buscar los valores posibles de $\mathrm{p}$ que hacen que las funciones de Bessel $J_{m}$ valgan cero. Dado que en la ec. (14), cuando q tiende a cero, $p=V$, se utilizan los valores encontrados de $p$, para obtener la frecuencia de corte de los modos $\mathrm{EH}$.

$$
\left.f_{c}\right|_{E H_{m n}}=\frac{p_{m n} c}{(2 \pi a)(N A)}
$$

1 Esta solución fue obtenida por medio del método numérico denominado "conducción débil". 
Los valores de $\rho_{m n}$ se consignan en la Tabla I.

Tabla 1. Raíces $\mathrm{P}_{\mathrm{mn}}$ para las cuales $J_{\mathrm{m}}(\boldsymbol{\rho})=0$ y se cumple la condición de corte para los modos $E H_{m n}, \rho \neq 0$.

\begin{tabular}{|c|c|c|c|c|}
\hline & $\mathbf{n = 1}$ & $\mathbf{n = 2}$ & $\mathbf{n = 3}$ & $\ldots$ \\
\hline $\mathbf{m = 1}$ & 2.405 & 5.520 & 8.654 & $\ldots$ \\
\hline $\mathbf{m = 2}$ & 3.832 & 7.016 & 10.173 & $\ldots$ \\
\hline $\mathbf{m = 3}$ & 5.136 & 8.417 & 11.620 & $\ldots$ \\
\hline$\ldots$ & $\ldots$ & $\ldots$ & $\ldots$ & $\ldots$ \\
\hline
\end{tabular}

Realizando un análisis similar, se obtienen los valores de las primeras raíces, consignadas en la Tabla 2.

Tabla 2. Raíces $\rho_{m n}$ para las cuales $J_{j}(p)=0$ y se cumple la condición de corte para los modos $E H_{1 n}$

\begin{tabular}{|l|c|c|c|c|c|}
\hline & $\mathbf{n = 1}$ & $\mathbf{n = 2}$ & $\mathbf{n = 3}$ & $\mathbf{n = 4}$ & $\ldots$ \\
\hline $\mathbf{m = 1}$ & 0 & 3.832 & 7.016 & 10.173 & $\ldots$ \\
\hline
\end{tabular}

Se puede proceder de forma similar para obtener los valores de las raíces para los demás modos de propagación. A continuación, se consignarán los valores en la Tabla 3 para facilitar la resolución de ejercicios ${ }^{2}$

Tabla 3. Condiciones para la frecuencia de corte normalizada $\left(V_{c}=\rho\right)$ de los modos de propagación en una fibra de índice escalonado

\begin{tabular}{|c|c|c|c|c|c|c|}
\hline \multirow[b]{2}{*}{$\mathbf{m}$} & \multirow{2}{*}{ modos } & \multirow{2}{*}{ condición } & \multicolumn{4}{|c|}{ Primeras raíces } \\
\hline & & & $\mathrm{n}=1$ & $n=2$ & $n=3$ & \\
\hline 0 & $\begin{array}{l}\mathrm{TE}_{\text {on }} \\
\mathrm{TM}\end{array}$ & $J_{d}(V)=0$ & 2.405 & 5.530 & 8.654 & \\
\hline 1 & $\mathrm{HE}_{1 n}$ & $J_{l}(V)=0$ & 0 & 3.832 & 7.016 & \\
\hline 1 & $\mathrm{EH}_{1 n}$ & $J_{l}\left(V_{d}=0\right.$ & 3.832 & 7.016 & 10.173 & \\
\hline \multirow{3}{*}{$\geq 2$} & \multirow{3}{*}{$\mathrm{HE}_{m n}$} & \multirow{3}{*}{$J_{m-2}\left(V_{d}\right)=0$} & 2.405 & 5.530 & 8.654 & $m=2$ \\
\hline & & & 3.832 & 7.016 & 10.173 & $m=3$ \\
\hline & & & 5.136 & 8.417 & 11.620 & $m=4$ \\
\hline \multirow{2}{*}{$\geq 2$} & \multirow{2}{*}{$\mathrm{EH}_{\mathrm{mn}}$} & \multirow{2}{*}{$J_{m}(V)=0$} & 5.136 & 8.417 & 11.620 & $m=2$ \\
\hline & & & & & & \\
\hline
\end{tabular}

2 La Tabla 3 proporciona información sobre la cantidad de modos que pueden coexistir simultáneamente en la fibra de índice escalonado a determinada frecuencia. 


\section{DISEÑO DEL PROGRAMA DE CÁLCULO}

Inicialmente, el programa captura los valores de los índices de refracción $n_{1}$ y $n_{2}$ para el núcleo y el revestimiento respectivamente, así como el valor del radio a del núcleo de la fibra y la cantidad de modos de propagación que se desean obtener. El objetivo es calcular el valor de la apertura numérica $N A$, la frecuencia de corte normalizada $V_{c}$ y la frecuencia de corte real $f_{c}$ para la cantidad de modos de propagación elegida por el usuario. Para el cálculo de $N A$, simplemente basta con reemplazar los valores de $n_{1}$ y $n_{2}$ en la ec. (19), ya que este es un valor fijo para todos los modos de propagación. En cuanto al cálculo de $f_{c^{\prime}}$ se partirá de la ec. (15), de la cual se obtiene:

$$
f_{c}=\frac{c V_{c}}{2 \pi a N A}
$$

Previamente se debe obtener los valores de $V_{c}$ para cada uno de los modos de propagación a partir de la Tabla III, y mostrarlos. Por último, a partir de estos valores, se obtienen las frecuencias de corte de los modos establecidos y se hace su representación. El entorno gráfico se muestra en la Figura 4. El programa cuenta con una sección de elección de parámetros, donde el usuario ingresa los valores solicitados $\left(n_{1}, n_{2} y\right.$ a), además de la posibilidad de escoger los modos para los cuales se desea realizar los cálculos.

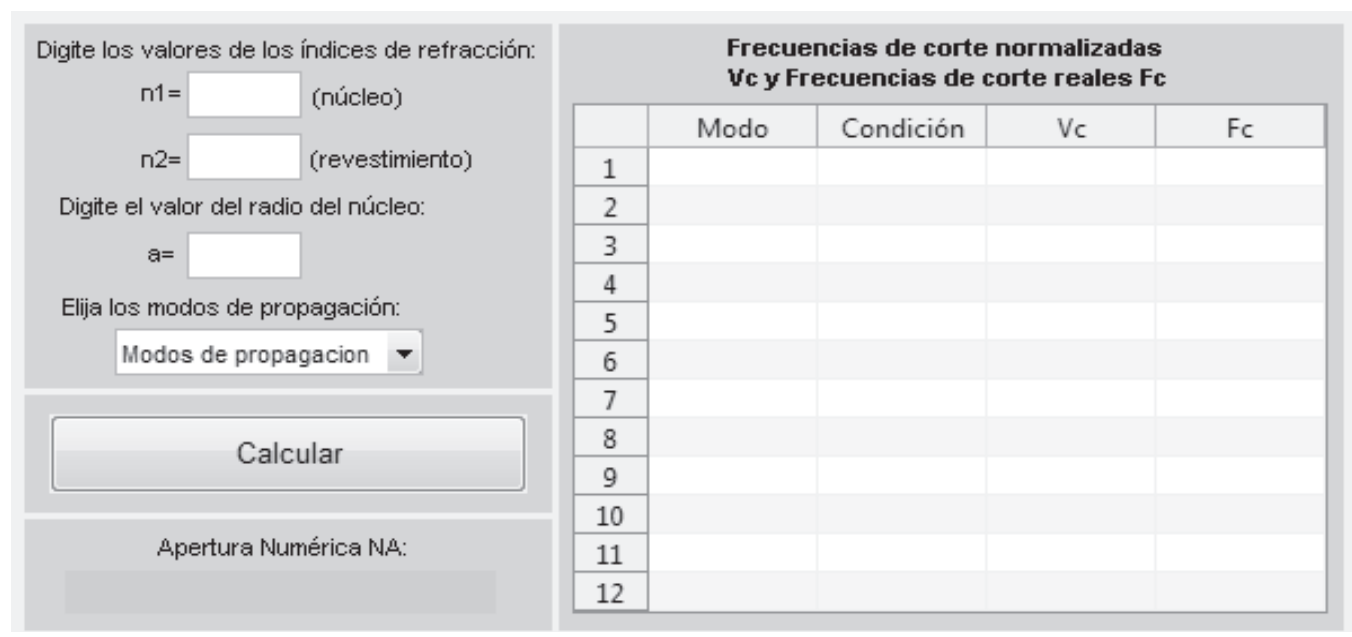

Figura 4. Entorno gráfico de la aplicación

Según esta selección, al presionar el botón calcular, el programa proporciona el valor de la apertura numérica y la condición para $J_{m}(V d$, la frecuencia de corte normalizada y la frecuencia de corte real para cada modo deseado. 


\section{RESULTADOS}

Se realizó la verificación de los resultados obtenidos con el programa mediante la comparación entre un ejercicio resuelto teóricamente y por medio del programa desarrollado ${ }^{3}$. El problema consiste en obtener la apertura numérica, las frecuencias de corte normalizadas y las frecuencias de corte real de los primeros 12 modos de propagación de una fibra óptica con índices de refracción 1.470 y 1.460 en el núcleo y revestimiento respectivamente, siendo el radio del núcleo de $8 \mu \mathrm{m}$.

\subsection{SOLUCIÓN TEÓRICA DEL PROBLEMA}

Para la solución de dicho ejercicio, inicialmente se utilizó la ec. (19) para obtener la apertura numérica.

$$
N A=\sqrt{n_{1}{ }^{2}-n_{2}{ }^{2}} \quad N A=\sqrt{(1.470)^{2}-(1.460)^{2}} \quad N A=0.1712
$$

Según la ec.(22), la frecuencia de corte real está dada por

$$
f_{c}=\frac{c V_{c}}{2 \pi a N A}
$$

Para lo cual, previamente ha debido obtenerse según la Tabla 3, los valores de las 12 primeras frecuencias de corte normalizadas (valores más bajos de $V_{c}$ a partir de cero). La Tabla 4 muestra la relación de los valores según el modo de propagación.

Tabla 4. Valores de Vc de los primeros 12 modos de propagación en la fibra óptica

\begin{tabular}{|c|c|}
\hline Modo & Valor de $\boldsymbol{V}_{\boldsymbol{c}}$ \\
\hline $\mathrm{HE}_{11}$ & 0 \\
\hline $\mathrm{TE}_{01^{\prime}}, \mathrm{TM}_{01}$ & 2.405 \\
\hline $\mathrm{HE}_{21}$ & 2.405 \\
\hline $\mathrm{HE}_{12}$ & 3.832 \\
\hline $\mathrm{EH}_{11}$ & 3.832 \\
\hline $\mathrm{HE}$ & 3.832 \\
\hline $\mathrm{EH}_{31}$ & 5.136 \\
\hline $\mathrm{HE}_{41}$ & 5.136 \\
\hline $\mathrm{TE}_{02}, \mathrm{TM}_{02}$ & 5.520 \\
\hline $\mathrm{HE}_{22}$ & 5.520 \\
\hline
\end{tabular}

3 Comparación basada en el ejercicio 7-1 del libro de Neri Vela [1] 
Con base en estos valores, se procede a obtener las frecuencias de corte reales así: Para el modo dominante $\left(\mathrm{HE}_{11}\right), f_{c}=0$

Para los modos $\mathrm{TE}_{01}, \mathrm{TM}_{01}$ y $\mathrm{HE}_{21}$, la frecuencia será:

$$
\begin{aligned}
& f_{c}=\frac{\left(3 \times 10^{8}\right)(2.405)}{2 \pi\left(8 \times 10^{-6}\right)(0.1712)} \\
& f_{c}=83.84 \times 10^{12} \mathrm{~Hz}
\end{aligned}
$$

Para $\mathrm{HE}_{12}, \mathrm{EH}_{11}$ y $\mathrm{HE}_{31}$ :

$$
\begin{gathered}
f_{c}=\frac{\left(3 \times 10^{8}\right)(3.832)}{2 \pi\left(8 \times 10^{-6}\right)(0.1712)} \\
f_{c}=133.58 \times 10^{12} \mathrm{~Hz}
\end{gathered}
$$

Para $\mathrm{EH}_{21}, \mathrm{HE}_{41}$

$$
\begin{gathered}
f_{c}=\frac{\left(3 \times 10^{8}\right)(5.136)}{2 \pi\left(8 \times 10^{-6}\right)(0.1712)} \\
f_{c}=179.04 \times 10^{12} \mathrm{~Hz}
\end{gathered}
$$

Para $\mathrm{TE}_{02^{\prime}} \mathrm{TM}_{02}$ y $\mathrm{HE}_{22}$

$$
\begin{gathered}
f_{c}=\frac{\left(3 \times 10^{8}\right)(5.520)}{2 \pi\left(8 \times 10^{-6}\right)(0.1712)} \\
f_{c}=192.43 \times 10^{12} \mathrm{~Hz}
\end{gathered}
$$

\subsection{SOLUCIÓN DEL PROBLEMA POR MEDIO DEL PROGRAMA DISEÑADO}

En primer lugar, se ingresan los datos requeridos: n1, n2 y a (Figura 5). Se eligen los modos de propagación deseados (Figura 6); para este caso, se seleccionó la opción "todos los modos". 
Digite los valores de los índices de refracción:

$\Pi 1=1.470$ (núcleo)
$\Pi 2=1.460$ (revestimiento)

Digite el radio del núcleo:

$a=8$ micrómetros

Figura 5. Ingreso de datos

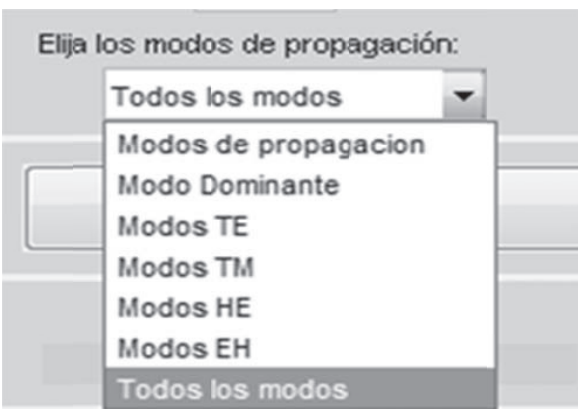

Figura 6. Elección del modo

Se procede a realizar el cálculo (Figura 7), y el primer resultado que se obtiene es el de la apertura numérica (Figura 8).

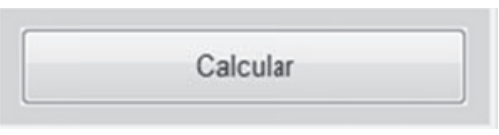

Figura 7. Calculo

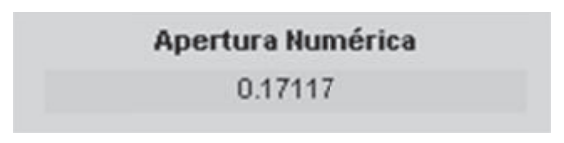

Figura 8. Resultado

En la Figura 9 se presenta la Tabla de resultados que se obtiene con el programa diseñado.

\begin{tabular}{|c|c|c|c|c|}
\hline & Modo & Condición & Vc & $\mathrm{Fc}(\mathrm{THz})$ \\
\hline 1 & HE11 & $\mathrm{J} 1 \mathrm{~V} \mathrm{c}=(0)$ & 0 & 0 \\
\hline 2 & TE01 & JoV $c=(0)$ & 2.4050 & 83.8557 \\
\hline 3 & TM01 & JoV $c=(0)$ & 2.4050 & 83.8557 \\
\hline 4 & HE21 & JoVc $=(0)$ & 2.4050 & 83.8557 \\
\hline 5 & HE12 & $J 1 V C=(0)$ & 3.8320 & 133.6113 \\
\hline 6 & EH11 & $J 1 V c=(0)$ & 3.8320 & 133.6113 \\
\hline 7 & HE31 & $\mathrm{J} 1 \mathrm{~V} \mathrm{C}=(0)$ & 3.8320 & 133.6113 \\
\hline 8 & $\mathrm{EH} 21$ & $\mathrm{~J} 2 \mathrm{~V} \mathrm{C}=(0)$ & 5.1360 & 179.0782 \\
\hline 9 & HE41 & $J 2 V c=(0)$ & 5.1360 & 179.0782 \\
\hline 10 & TE02 & JOVC $=(0)$ & 5.5200 & 192.4672 \\
\hline 11 & TMO2 & JoV $c=(0)$ & 5.5200 & 192.4672 \\
\hline 12 & HE22 & JoV $c=(0)$ & 5.5200 & 192.4672 \\
\hline
\end{tabular}

Figura 9. Resultados tabulados

\section{CONCLUSIONES}

Se presenta el diseño de una aplicación en Matlab para calcular los valores de apertura numérica, frecuencias de corte normalizadas y frecuencias de corte real en fibras ópticas de índice 
escalonado. Tomando en consideración que los mismos cálculos efectuados de manera manual al realizarlos en una calculadora o en el ordenador, implican pasos y operaciones muy laboriosas, ya que inicialmente se debe hallar el valor de la apertura numérica, verificar las condiciones para $J_{m}(V d$, elegir los modos en los cuales se produce propagación con base en las frecuencias de corte normalizadas proporcionadas por los valores de las funciones de Bessel, para finalmente consignarlos en una tabla que dé claridad sobre el problema, se proporciona a estudiantes y docentes una valiosa herramienta que simplifica notoriamente el problema. Surge de este proyecto, la posibilidad de hacer una ampliación del programa de tal forma que permita, a partir de una o unas frecuencias de corte requeridas, permita obtener los valores de los índices de refracción y el radio del núcleo para la construcción de una fibra óptica.

\section{REFERENCIAS BIBLIOGRÁFICAS}

[1] Couch León, (2009). Sistemas de comunicaciones digitales y analógicos. México, Prentice Hall, 600 p.

[2] Vela Nery R., (2004). Líneas de transmisión. México, McGraw-Hill, pp. 371-412.

[3] España, M., (2005). Comunicaciones ópticas: Conceptos esenciales y resolución de ejercicios. Madrid, Ediciones Díaz de Santos.

[4] Grassi Marangione F., (2007). Modelo simulink para redes de comunicaciones ópticas multiservicio. Ortega Tamarit, B. dir. ; Mora Almerich, J. dir. 44 p.

[5] Salleg J. y Torres C., (2006). Modos de propagación en una fibra óptica de índice de perfil escalonado. En: Revista de la Sociedad Colombiana de Física, Vol. 38.

[6] Pastor Abellán, D. (2008). Modos de propagación linealmente polarizados en fibras ópticas. Universitat Politècnica de València. Escuela Técnica Superior de Ingenieros de Telecomunicación - Escola Tècnica Superior d’Enginyers de Telecomunicaciónes. 
